\title{
Variaciones sobre el concepto de Ética ${ }^{1}$
}

\author{
Variations about ethics concept
}

\author{
Pérez Oliva, Elizabeth \\ Doctara en Cirumia Dental LEFS 1994 \\ Master en Administración y Docencia Uniressilaria UEES 2001 \\ Iniestigadora de la Dirección de Inzestigación de la LEES. \\ E-mail: enzabethotiza2002@gmailcom \\ Velasco, José Armando \\ Iic on Nitrición y Dietstica de Linuersidad de San Carlos, Guatemala \\ Profesar Liniemitano nizel Maestria UTG, \\ egesalo de Maestria en Dorencia Lnizersitara \\ Cardinadon de la Escuela de. Nutrición de la LEES. \\ E-mait: armando.vetaseo@urees.edu.si
}

\section{Resumen}

$A$ to largo de la historia, pensadores han dado ideas y teorías sobre la Ética, pero todos concuerdan en que es una parte de la Filosefá que trala de los actos morales, normas de conducia sabre el bien y el mal en el ser humano; término desarrollado a trazés del tiempo. Desde que tos hombres vizen en comunidad, la regulación moral de la conducta ha sido necesaria para el bienestar colectivo. Aunque los distintos sistema morales se establecian sobre pautas arbitrarias de conducla, evolucionaron a veces de forma irracional, a partir de que se ziolaran los tabúes religiosas o de conductas que primero fueron hábito y luego costumbre, o asi mismo de leyes impuestas por lideres para prevenir desequilibrios en el seno de la tribu. El término moral nos dice también. cómo hemos de comportarnos de manera que obrando de una determinada forma y seguin un determinado código moral, vayamos dando una determinada personalidad o forma de ser a nuestra vida. Segin nuestras costumbres y comportamientos damos forma a nuestra vida y adquirimos nuestro propio y particular modo de ser. Ese carácler o modo de ser se adquiere por medio de una serie de hábitos y costumbres. Antiguamente se consideraba que los dos términos se equivalian pero hoy se considera que la moral designa una serie de normas acepladas por un grupo social o establecido por un moralista. La palabra Élica es una expresión más técnica dentro de la Filosofia y da lugar a un sistema de principios filosóficos que son la base de esas normas.

Palabras clawe: Etica, Moral, normas, costumbres.

\section{Abstract}

T hroughout histon, thinkers have provided ideas and theories about Ethics, but all agree that it is a part of the Philosophy that deals with the moral acts, norms of conduct about the good and the bad in the human being; term developed through time. Ever since the men live in communit; the conduct moral regulation has been necessary for the community well-being. Although the different moral systems were established on arbitrary guidelines of conduct, they evolzed sometimes irrationally, ever since the violation of religious or conduct laboos that first were habits and then customs, as well as the lawes imposed by leaders to prevent imbalances inside the tribe. The moral term also tells us how we are to betave so that acting in a determined way and according to a certain moral code; we are molding a certain personality or zeay of being to our life. According to our customs and behaviors we mold our life and we acquired our oum and particular way of being That character or way of being is acquired by means of a series of habils and customs. Formerly it was considered that both terms were equivalent but loday it is considered that the moral designates a series of nurms accepled by a social group or established by a moralist. The w:ord Eithics is a more technical expression into Philosophy and leads to a system of philosophical principles that are the base of those norms.

Key words: Ethics, Moral, norms, customs. 


\section{Ética como disciplina filosófica.}

La Ética es una disciplina filosófica porque tiene su propio objeto de estudio; por lo tanto es una ciencia que tiene un conjunto sistemático de conocimientos racionales y objetivos acerca del comportamiento humano moral, y no es simplemente un capítulo de la Filosofia; desde este punto de vista es importante preguntarnos ¿qué es la Fỉosofia?

\section{¿Qué es la Filosofia?}

La definición de Filosofía ha cambiado considerablemente durante el curso de la historia de tal manera que no puede encontrarse una definición única y universal sino tantos conceptos como doctrinas filosóficas han aparecido en la historia, a tal grado que el filósofo J.M. Brochenski a la pregunta ¿Qué es propiamente la Filosofia? Responde: "lastimosamente ésta es una de las cuestiones filosóficas más difíciles" (Escobar Valenzuela, 2006)

El diccionario de la Real Academia de la Lengua Española define el concepto de Filosofía de la forma siguiente: "Conjunto de saberes que busca establece, de manera racional, los principios más generales que organizan y orientan el conocimiento de la realidad, así como el sentido del obrar humano". La Filosofía es un intento de dar una explicación a la realidad de manera lógica y racional y al tener ésta comportamientos multidisciplinarios, surgieron otras ciencias que buscan explicar la diversidad del comportamiento humano dando lugar a la ciencia de la Etica.

La Ética es una disciplina filosófica. De acuerdo con el tipo de problemas que abordan las disciplinas o ramas de la Filosofia se han divido en disciplinas teóricas o gnoseológicas y disciplinas prácticas (Escobar Valenzuela, 2006).

Las disciplinas teóricas o gnoseológicas giran en torno a los problemas cognoscitivos. Aquí se pucde ubicar la Teoría del Conocimiento o Gnoseología (de gnosis: conocimiento y logos: (ratado o estudio) cuyos planteamientos se refieren a los modos, clases, esencia, posibilidad, validez y origen del conocimiento humano; a la Lógica que se propone investigar los elementos y estructuras normales de los sistemas de enunciados, y a la Filosofia de la Ciencia (llamada en ocasiones, Epistemología), que investiga la estructura, el fundamento y los métodos desarrollados por sistemas científicos.

Las disciplinas prácticas se refieren a la acción humana. Además de preocuparse por temas eminentemente teóricos, denominados abstractos, la Filosofia centra su atención en los principios, formas y sentidos de la praxis humana, en la naturaleza de la conducta individual y colectiva, sus fines, normas y valores en el problema en cl que son el deber, la libertad, la obligatoriedad, las virtudes morales, etc.

Dentro del conglomerado de disciplinas prácticas de Filosofía podemos mencionar la Ética, la cual estudia la conducta moral del hombre en la vida social. La Ética, en cuanto a disciplina filosófica, queda ubicada como una disciplina práctica de la Filosofía de acuerdo con su tema de estudio que es la moral, conforme a la cual el hombre modela su existencia.

La clasificación antes mencionada se esquematiza en el cuadro siguiente:

\begin{tabular}{|c|c|c|}
\hline \multirow{10}{*}{$\begin{array}{l}\text { Disciplinas } \\
\text { filosóficas }\end{array}$} & \multirow{3}{*}{$\begin{array}{c}\text { Teóricas o } \\
\text { gnoseológicas }\end{array}$} & Teoría del conocimiento \\
\hline & & Lógica \\
\hline & & Filosofia de la Ciencia \\
\hline & \multirow{7}{*}{ Prácticas } & Axiológicas \\
\hline & & Etica \\
\hline & & Estética \\
\hline & & Filosofía del Derecho \\
\hline & & Filosofia Social y Politica \\
\hline & & Filosofia de la Educación \\
\hline & & Filosofía de la Religión \\
\hline
\end{tabular}

\section{Definición etimológica de Ética.}

Como todo saber, la Ética puede ser analizada etimológicamente investigando sus raíces linguiísticas.

El término Ética se deriva de la palabra griega ethos "costumbres" (Velásquez), "modo de ser" o "carácter"( Sánchez Vázquez, 1969), "carácter" (Roman Maestre).

Escobar Valenzuela menciona que la acepción más conocida y difundida del vocablo ethos se presenta a partir de Aristóteles, ligada a un conocimiento llamado precisamente Ética. Según esta acepción ethos significa temperamento, carácter, hábito, modo de ser. De acuerdo con el significado etimológico, Ética seria una teoría o un tratado de los hábitos y las costumbres.

Una característica del ethos, entendido como conjunto de hábitos y maneras de ser del hombre, es su sentido no natural. En efecto el ethos, scgún la tradición gricga implica una serie de costumbres adquiridas por hábito y no innatas; el ethos significa una conquista del hombre a lo largo de su vida. En este sentido se dice que el ethos constituye una "segunda naturaleza"; se trata de un conjunto de hábitos de los cuales el hombre se apropia, modificando su naturaleza. Este concepto de la segunda naturaleza del hombre lo afirma Sánchez Vázquez en su libro "Ética" (1969).

\section{Definición de Ética.}

Sánchez Vázquez (1969) la define como la teoría o ciencia del comportamiento moral de los hombres en socicdad, o sea, es ciencia de una forma específica de conducta humana.

Cotidianamente cuando se habla de Ética se está orientando hacia la comprensión del bien y del mal, para poder medir si una acción humana es correcta o incorrecta a la luz de los valores morales de la cultura en la que se vive, así por ejemplo: ¿Quién no 
aprecia que alguien que encuentra un objeto de su propiedad se lo devuelva? Cuando emitimos un juicio ético de un comportamiento valoramos la maldad o bondad de una "acción". En otras ocasiones, lo que juzgamos es a la "persona" que realiza este tipo de acciones y hablamos de "sinverguenza" para calificar a alguien que actúa de mala fe, o de una bellísima persona para expresar la calidad humana de alguien.

En este sentido, la Ética hace referencia a la calidad humana de las personas y de sus acciones o como lo entendieron los clásicos, a su excelencia. En una primera aproximación al concepto, cabe decir que un comportamiento se califica como Ética, precisamente cuando contribuye al desarrollo de las cualidades propias del ser humano o, lo que es lo mismo cuando hace el individuo mejor persona, cuando su calidad humana crece (Guillen Parra, 2006)

\section{La Ética concebida como ciencia.}

El carácter científico de la Ética responde a la necesidad de un tratamiento científico de los problemas morales. De acuerdo con este tratamiento la Ética se ocupa de un objeto propio: El sector de la realidad humana que llamamos moral, constituido como un conjunto peculiar de hechos o actos humanos. Como ciencia la Ética parte de cierto tipo de hechos tratando de descubrir sus principios generales. En este sentido, aunque parte de datos empíricos, o sea, de la existencia de un comportamiento moral efectivo, no puede mantenerse a nivel de una simple descripción o registro de ellos, si no que los trasciende con sus conceptos, hipotesis y teorías. En cuanto conocimiento científico, la Ética ha de aspirar a la racionalidad y objetividad mas plenas, y a la vez a proporcionar conocimientos, sistemáticos, metódicos y hasta donde sea posible verificables (Sánchez Vásquez, 1969).

La Ética es Filosofia Moral, es decir, reflexión críticoracional sobre la moral. No obstante, la Ética no recopila las distintas morales de la historia en un afán meramente descriptivo o recopilatorio sino que se interroga sobre si las morales son legítimas, si tienen razón de ser y si dicha razón es correcta. Las morales son vivencias cotidianas, hechos estudiados por la Psicología, Historia, Sociología, pero la Filosofía Moral, la Ética, no se limita a constatar los hechos sino que se pregunta, más allá de su vigencia por su validez. (Roman Maestre, 2007).

La Ética es teoría, investigación o explicación de un tipo de experiencia humana, o forma de comportamiento de los hombres: el de la moral, pero considerado en su totalidad, diversidad o varicdad. El valor de la Ética como Teoría está en lo que explica y no en prescribir o recomendar con vista a la acción en situaciones concretas.
La Ética es la ciencia de la moral, es decir de una esfera de la conducta humana, no hay que confundir aquí la teoría con su objeto: El mundo moral.

\section{Método de la Ética.}

Todas la ciencias se caracterizan por tener un método propio que le da su carácter téórico, pues hay una correlación entre teoria y método ya que toda teoría implica necesariamente un método, significa entonces que no puede haber una ciencia sin método de investigación, porque el carácter normativo deriva de su objeto de estudio, mientras que el carácter teórico deriva del método.

La palabra método viene del griego "metă" que significa fin y de "odos" que significa camino, es decir" el camino que conduce al fin", en general se denomina método al conjunto de procedimientos adecuados para obtener un fin; cn cl caso específico de la Ética este fin es el conocimiento de la moral. Debe buscarse entonces un método que se acomode al objeto de la Ética, por tanto el método de la Ética debe tener en cuenta el aspecto humano social $\mathrm{e}$ histórico inherente a su campo de investigación.

Al revisar la historia de la Filosofia se llega a la conclusión de que no hay un criterio unánime de cual es el método propio de la Ética, porque históricamente cada pensador o filósofo a propuesto su propia metodología.

Escobar Valenzuela en su texto Ética, Introducción a su problemática y su materia, menciona que históricamente la Ética ha utilizado al menos tres métodos para explicar su teoría, los cuales son los siguientes:

1. Método inductivo. Consiste en partir de lo particular hacia lo general; sus partes son: la observación, la comparación y la experimentación de los distintos hechos concretos de la vida humana para formular normas morales de validez universal. Generalmente este método es utilizado por ciencias experimentales Biología, Química, Fisiología, etc.)en ciencias donde intervienen fundamentalmente la observación y la experimentación. Sin embargo, Aristóteles observa las costumbres de su época para derivar que era el sumo bien aquello a que todos los hombres aspiran (la felicidad). Este procedimiento de Aristóteles puede considerarse ya como una aplicación del método inductivo en la Ética.

Otro ejemplo de procedimiento inductivo en la historia de la época se encuentra en aquellas tendencias que se han agrupado bajo el nombre de Ética empírica, es decir, de la observación de las costumbres o fenómenos que pasan en la realidad. De tal manera que la observación frecuente que los débiles cran subyugados por los fuertes dio origen a la norma de conducta "el 
poderoso debe someter al débil" cumpliendo así una ley natural o que "el derecho se funda en cl poder". En los empiristas la Ética es ciencia de lo que es, mas no de lo que debe ser.

2. Método deductivo. La deducción es el procedimiento o método que parte de lo general para llegar a lo particular. Consiste en que de una ley ya establecida se deducen normas de conducta particular. Este método es el propio de las ciencias formales (Lógica, Matemática). Se podría decir que d procedimiento deductivo ha sido la manera de pensar propia de las éticas especulativas y tradicionales. Estas éticas tratan de deducir sus conceptos éticos a partir de una idea inalterable de la naturaleza humana.

La Ética como toda ciencía posee un método por medio del cual se tenga un conocimiento profundo de la conducta humana.

Los pasos del método, ya sea inductivo o deductivo, propuestos por Aguayo R. en su monografia "Ética Profesional" son los siguientes:

- Observación. Este paso también es propio del método científico. La observación no solo consiste en acercarse al hecho real y percibir a través de los sentidos en forma penetrante y amplia.

- Evaluación. A partir de la percepción del acto por medio de la observación, se emiten un juicio de valor moral, es decir tratar de catalogar el acto observado dentro de las categorías morales previamente establecidas estudiadas como pueden ser: reprobable, honesto, obligatorio, bueno, amable, recomendable, etc. Es necesario que existan matrices de valoración moral para así poder catalogar con más detalle el acto estudiado.

- Percepción axiológica. En este aspecto se trata de descubrir en forma personal los valores que todavía no se ha sido capaz de descubrir o percibir en este acto. Una vez hecho esto podemos darle un valor al acto estudiado de acuerdo a una escala de valores.

3. Métodos filosóficos. Los métodos deductivos e inductivos pertenecen a los métodos científicos. La Filosofia a partir de sus propias concepciones, crea los métodos filosóficos basándose en la Lógica $y$ es asi como surgen los métodos: Fenomenológicos, Crítico Trascendental y el Dialéctico que tienen aplicación en el estudió de la Etica.

El método fenomenológico fue creado por el filósofo alemán Edmund Husserl (1859-1938). La Fenomenología significa estudio o descripción de los fenómenos (morales, históricos, religiosos, etc) para determinar su eidos o esencia. Este método pucde ser útil en la Ética en dos sentidos: Directamente, analizando fenómenos morales para determinar sus rasgos esenciales, e indirectamente, sirviéndose de otros análisis fenomenológicos de campos colindantes con la moral.
El método crítico trascendental fue creado por Emmanuel Kant (1724-1804). Este método consiste en proyectar la atención no sólo sobre los objetos mismos sino sobre el saber que nos da la cultura indagando las bases o elementos a priori del conocimiento.

El mélodo dialéctico. Sócrates también lo deno-minó mayéutica o arte de dar a luz la verdad por medio del dialogo. En general la dialéctica consiste en afirmar que el ser o la historia se desenvuelven por el paso de una tesis a una antítesis y de esta oposición a una sintesis.

\section{Objeto de la Ética.}

La moral no es ciencia sino objeto de la ciencia, en este sentido es estudiada e investigada por la ciencia.

La Ética no es la moral y por ello no puede reducirse a un conjunto de normas y prescripciones; su misión es explicar su moral efectiva y en este sentido puede influir en la moral misma.

Su objeto de estudio lo constituye un tipo de actos humanos: Los actos conscientes y voluntarios de los individuos que afectan a otros, a determinados grupos sociales o a su conjunto.

Ética y moral se relacionan pues la moral es el objeto de estudio de la Ética. Una y otra palabra, mantienen así una relación, que no tenían propiamente en sus origenes ctimológicos, porque el vocablo moral procede del latin mos o mores que significa costumbre o costumbres, en el sentido de conjunto de normas o reglas adquiridas por hábitos. La moral tiene que ver así con el comportamiento adquirido o modo de ser conquistado por el hombre.

\section{Moral. \\ Definición.}

Velásquez (2007) en sus disertaciones teóricas define la Moral como un "conjunto de normas que regulan el comportamiento de los individuos y grupos."

Sánchez Vázquez (1969) la define como un conjunto de normas aceptadas libre y conscientemente que regula la conducta individual y social de los hombres, la cual coincide con la citada por Escobar Valenzuela.

Román Maestre dice que es la vivencia cotidiana de adhesión a valores, principios, normas, a partir de los cuales juzgamos los actos como correctos/incorrectos.

La moral entonces es un conjunto de reglas que la sociedad exige que un hombre observe dentro de ella. Un hombre moral es aquel que vive en concordancia con las costumbres de su sociedad; en caso de infringir las normas el hombre será marginado de la sociedad. Podemos decir tambien que la moral es un conjunto de normas y formas de vida a través de las cuales $\mathrm{cl}$ hombre aspira a realizar el valor de lo bueno. Para adentrarnos en los problemas de la Moral, 
nos señala nuestra propia experiencia que, en determinadas circunstancias, cxpresamos valorizaciones morales. Los contenidos de nuestros juicios se refieren a categorías opuestas: de bien o mal.

Así hablamos de actos nobles, heroicos, desinteresados, los que están en la esfera del concepto de "bien". Pero, hablamos de actos infames, egoístas, los que están en la esfera del concepto de "mal".

En el primer caso, hablamos de actos morales positivos; en el segundo, de actos morales negativos. Así, por ejemplo: a un asesino lo tratamos de inmoral: a una persona que sacrifica su vida para salvar a otro la tratamos de héroe, pues este sacrificio es de gran calidad moral. Ello nos pone en evidencia que nuestra valoración tiene un carácter objetivo, es decir, que se rige por el objeto que verificamos. En nuestro caso, el asesinato, y por otro lado, el heróismo. Dicha objetividad nos permite formar juicios morales de validez universal: "El asesinato es una inmoralidad independiente de su implicación penal".

Las características fundamentales que presenta la moral son:

a) Es normativa, constituida por las normas o reglas de acción e imperativos que enuncian algo que debe ser. En otras palabras la norma es obligatoria

b) Es fáctica, orientada al plano de los hechos morales, constituida por ciertos actos humanos que se dan efectivamente, es decir que son, independientemente de cómo estimemos que debieron ser.

Al plano normativo pertenecen las reglas que postulan determinado tipo de actuar: "Ama a tu prójimo como a ti mismo", "Respeta a tus padres", "No mientas", "Saluda al llegar a un lugar", etc.

$\mathrm{Al}$ plano de lo fáctico corresponde siempre acciones concretas: el acto por el que Pedro se muestra solidario con Mario.

A la luz de lo expresado anteriormente se puede concluir que en la realidad existen normas específicas de comportamiento, es decir la teoría de la Moral, lo que da origen a lo que comúmmente llamamos moral pero los hechos concretos del hombre, es decir la moral aplicada, es lo que se conoce como moralidad.

Las normas están determinadas por la cultura y es por eso que existen varias normas, lo que le da el concepto de relatividad, es decir, lo bueno en una cultura, no necesariamente lo será en otra. Roman Macstre (2007) menciona que la moral inculca hábitos (hexis), a partir de la repetición y la vivencia cotidiana. Muchas veces los valores, normas, por los que las personas se rigen son inconscientes. Es posible que haya quien se adhicra a valores sin juzgarlos, y a eso es a lo que llamamos pre-juicio.
Relaciones y diferencias entre Ética y Moral.

Como conclusiones de esta distinción entre Ética y Moral se puede afirmar:

1. La Moral pregunta qué debo hacer (hábitos, costumbres). La Ética pregunta por qué debo hacerlo, se situa así en un nivel superior de profundidad. El por qué es una pregunta por la fundamentación de las costumbres, de las morales, es una pregunta por su razón de ser.

2. Etimológicamente la raíz latina mos alude a la costumbre, a lo que es normal (conforme a norma), habitual. Lia palabra griega ethos quiere decir carácter, personalidad. Si apreciamos las etimologías, podemos decir que la Ética exige en su reflexión crítica a las morales, es decir que las normas/costumbres sirvan a la forja del carácter y de la personalidad.

3. La Ética estudia las morales para legitimarlas (comprobar su validez), por lo que no cualquier Moral es legitima, aunque la gente siga esa moral (se constate su vigencia). La razón de que una Moral sea auténtica o no es que coadyuve a la forja del carácter, para lo cual toda moral ha de respetar la universalización, la autonomía y la necesidad u obligación con que se presentan los preceptos.

4. La reflexión crítico-racional ha de transformarse en moral, costumbre. La Ética tiene que volverse un hábito. Esta Ética misma es fruto de un proceso que es un progreso moral. No se llega a tener el hábito crítico racional sino gracias a una evolución de la concicncia moral.

\section{Conclusiones}

Las palabras Ética y Moral suelen utilizarse en nuestro medio como sinónimos; pero al revisar la literatura, se muestran claras diferencias entre una y la otra.

La Ética es Filosofia Moral, es decir una reflexión crítico-racional sobre la moral.

La Ética es el estudio filosófico y cicnúfico de la Moral. La ética es teórica, mientras que la moral es práctica. Las dos se refieren a normas de comportamiento.

La Moral es normativa, porque establece las normas que determinan lo que es el bien y lo que es el mal. En otras palabras, la Moral establece lo que se debe y lo que no se debe hacer. Lo que se permite y lo que se prohíbe. También se le conocc como la Conciencia Moral

La Moral formula principios generales. Según estos principios clasifica y juzga todos los hechos particulares que son propios de su campo.

La Ética se finca en la razón, y depende de la Filosofia. La Moral se apoya en las costumbres, y la conforman un conjunto de elementos normativos, que la sociedad acepta como válidos. 


\section{Fuentes de informacion consultadas}

1. Aguayo R., Paulino. La ética profesional. Universidad Nacional de Pilar - Ayolas - Paraguay Curso de Postgrado de Didáctica Universitaria

http: / /www monografias.com/lrabajos 47/eticaprofesional/eticaprofesional2. shiml

2. Diccionario de la Real Academia de la Lengua Española. hutp://buscon.rae.es/draeI/SroliConsulla?'TIPO BUS=3 LEMA = filosofia Consultado el 22 de Septiembre de 2007.

3. Escobar Valenzuela, Gustazo: Etica, introducuón a su problemática y su materia McGraze-Hill Interamericana. 2005. Edición: 5 a. ed. México D.E

4. Escobar Valenzuela, Gustavo. Etica. McGraw-Hill Interamericona. 2001. México D.F

5. Guillen Parra, Manuel. Etica en las organizaciones, Construyendo confianza. Pearson Educación S,A. Madrid. 2006.
6. Sänchez Vázquez, Adolfo. Ética. Editonal Grijalbo, S.A. de C.V. Méxzo, D.F 1969

7.Rychlowsk .Bruno. Ética: Conceptos de Ética y Moral. hutp://wwew apocatastasis.com/moral-etica-conceptos.pht

8. Velásquez, Humberto. Clase magistral y discusiones de la Cátedra de Teoria del Conocimiento de la Maestría om Melodologia de la Investigación Cientifica de la Universidad Evangélica de El Salvador. septiembre de 2007.

9. Weber, Max. La Elica Protestante y el espiritu del capitalismo. Primera Edición. Premia Edilora, México, D.F 1979

10. Roman Maestre, Begoña El problema de los juicios morales. La distinción entre Etica y moral. Curso de Iniciación y Fundamentacion Bioética. Primer Capitulo Mod, I hitp://campus.ibbioetica.org/ Consultado el 19 de septiembre de 2007

11. Valverde Aragundi, Víclor: La conciencia Morat. Consultado el 26 de septiembre de 2007. Disponible en: waremonografias com/trabajas $5 /$ commo/commoshitm/3nonosearh 